\title{
Evaluation of Mesh Simplification Algorithms using PolyMeCo: A Case Study
}

\author{
Samuel Silva ${ }^{a}$, Frutuoso Silva ${ }^{b}$, Joaquim Madeira $^{a, c}$ and Beatriz Sousa Santos ${ }^{a, c}$ \\ ${ }^{a}$ Institute of Electronics and Telematics Engineering of Aveiro, University of Aveiro, \\ Aveiro, Portugal \\ ${ }^{b}$ IT - Networks and Multimedia Group / Dep. of Computer Science and Engineering, \\ University of Beira Interior, Covilhã, Portugal \\ ${ }^{c}$ Dep. of Electronics, Telecommunications and Informatics, University of Aveiro, \\ Aveiro, Portugal
}

\begin{abstract}
Polygonal meshes are used in many application scenarios. Often the generated meshes are too complex not allowing proper interaction, visualization or transmission through a network. To tackle this problem, simplification methods can be used to generate less complex versions of those meshes.

For this purpose many methods have been proposed in the literature and it is of paramount importance that each new method be compared with its predecessors, thus allowing quality assessment of the solution it provides. This systematic evaluation of each new method requires tools which provide all the necessary features (ranging from quality measures to visualization methods) to help users gain greater insight into the data.

This article presents the comparison of two simplification algorithms, NSA and QSlim, using PolyMeCo, a tool which enhances the way users perform mesh analysis and comparison, by providing an environment where several visualization options are available and can be used in a coordinated way.
\end{abstract}

Keywords: Polygonal Meshes, Quality Evaluation, Visualization.

\section{INTRODUCTION}

Polygonal meshes are widely used in many application scenarios like CAD/CAM, video games, cultural heritage ${ }^{1}$ and physically-based modeling. ${ }^{2}$ In many of these applications the original meshes (obtained, e.g., using 3D scanners) are very complex and do not allow proper visualization and interaction (e.g., in low resources systems like $P D A s$ ) or their transmission through a network. In these cases the solution can be mesh simplification.

Many simplification algorithms have been proposed in the literature ${ }^{3}$ but only a few studies compare those methods with each other, helping the user choose the proper method for a specific application (examples are those of Cignoni et al. ${ }^{4}$ and Rogowitz et al. ${ }^{5}$ ). For the purpose of mesh comparison, some metrics (e.g., geometric and normal deviations) and tools have been proposed in the literature. How these metrics estimate user perceived quality is still an unanswered question, but some authors as Watson et al. ${ }^{6}$ and, more recently, Silva et al. ${ }^{7,8}$ and Santos et al. ${ }^{9}$ who compare simplification methods using observer studies, aim to produce guidelines which help users understand not only the physical implications of a particular metric but how it can be used to estimate perceived quality.

Meanwhile, mesh comparison tools must also go a step forward, providing a user-friendly integrated environment with several visualization options, focusing on turning mesh comparison into a systematic task available not only to researchers developing new algorithms but also to those who use them and wish to evaluate their results.

In this article the comparison of a recent simplification method developed by the second author, $N S A,{ }^{10}$ with the widely used method QSlim $^{11}$ is presented. This comparison is performed using PolyMeCo, ${ }^{12}$ a tool for mesh

Further author information: (Send correspondence to Samuel Silva.)

E-mail: sss@ieeta.pt, Telephone: +351 234370500 
analysis and comparison. The main goal is to assess if NSA provides better results than QSlim while exploring the functionalities provided by PolyMeCo, thus illustrating a typical application for this kind of tool.

On the following sections a brief description of the used simplification algorithms and of mesh comparison tools previously presented in the literature is given. Then, the main features of PolyMeCo, the tool used to perform the comparison, are described. Afterwards, a case study is presented which shows the results obtained for the comparison between the simplification algorithms and how it was performed using PolyMeCo. Finally, some conclusions are presented.

\section{SIMPLIFICATION ALGORITHMS: QSLIM AND NSA}

There are several types of algorithms for polygonal mesh simplification (see Cignoni et al., ${ }^{4}$ Puppo et al. ${ }^{13}$ and Luebke et al. ${ }^{3}$ ). We used the QSlim $^{11}$ and $N S A^{10}$ algorithms to evaluate the different quality criteria for generated meshes. QSlim is freeware and available from http://www.cs.cmu.edu/ garland, which has allowed applying both algorithms to the same models. Both algorithms simplify a mesh by iteratively collapsing edges into vertices, i.e., using the edge collapse operation.

The edge collapsing operation is standard. The main difference between the various edge collapsing-based simplification algorithms is the criterion used to choose the next edge to collapse. A different criterion implies different mesh quality, as well as a distinct processing time. Generally, all simplification algorithms make a trade-off between speed and the quality of the resulting mesh.

QSlim follows a geometric criterion that is based on the minimization of the error associated with each new vertex. This error is defined as the sum of the squared distances to the set of planes surrounding the pair of the original collapsing vertices. Thus, this algorithm produces simplified meshes with a very good geometric quality since it minimizes the error associated with each new vertex.

On the other hand, the NSA algorithm follows a geometric criterion which implies that the region around the collapsing edge be nearly coplanar. An edge is only collapsed if the variation of the face normals around the target edge is within a given tolerance $\varepsilon$. The value of $\varepsilon$ is the threshold for the angle between the current normal and the new normal after the edge collapsing operation.

$N S A$ is faster than QSlim without degrading mesh quality too much. ${ }^{10}$ In general, it provides good results in terms of shape preservation, time performance, and mesh quality. Note that NSA was primarily developed for time efficiency purposes and mesh quality was not a priority goal (but it is also achieved).

\section{MESH COMPARISON TOOLS}

Mesh comparison is usually carried out with the help of dedicated software tools providing the user with numerical data (e.g., minimum, mean and maximum difference values) and visual information (e.g., coloring a model according to the difference values measured at each vertex), allowing the user to choose among several difference measures. A few such tools, which allow polygonsl mesh feature evaluation and comparison, are described in the literature.

Metro, developed by Cignoni et al., ${ }^{14}$ allows both numerical and visual mesh comparison. Among the numerical values provided is data about the input mesh characteristics (number of vertices and faces, surface area, mesh volume, etc.), the minimum and maximum distances between two given meshes and their difference in volume. It is also possible to view a model colored according to the results obtained. The Hausdorff distance between two meshes can also be computed.

The tool described by Zhou et al. ${ }^{15}$ introduces some additional measures (namely, surface curvature) and uses several visualization techniques, including side-by-side viewing of the compared models and difference results, box-glyphs and animations.

$M E S H$, developed by Aspert et al., ${ }^{16}$ uses the Hausdorff distance to measure the difference between two mesh models. It provides several numerical values, namely the main characteristics of the input meshes and the minimum, mean, maximum and RMS values of the computed difference measures. It is also possible to view a model colored according to the obtained results. 
Finally, Roy et al. ${ }^{17}$ describe a tool called MeshDev which allows the computation of geometric, normal and other mesh attribute deviations such as color or texture. Similarly to the other tools, it provides several numerical values characterizing the input meshes and the obtained results. It is also possible to view the models colored according to the computed deviations.

\section{POLYMECO}

Although a few mesh comparison tools have been proposed in the literature, they still lack some features which would allow the mesh evaluation process to become a more systematic task applied in a wider range of situations. For example, in an evaluation process it is often necessary to compare and evaluate many models. So, it is desirable that the tool used to perform such an evaluation can be applied to all the models simultaneously, instead of evaluating each model at a time. This tool must also provide visualization options which allow users to gain additional insight into the data obtained, thus freeing them from using other tools to perform the visualization, which would make the evaluation process more time consuming.

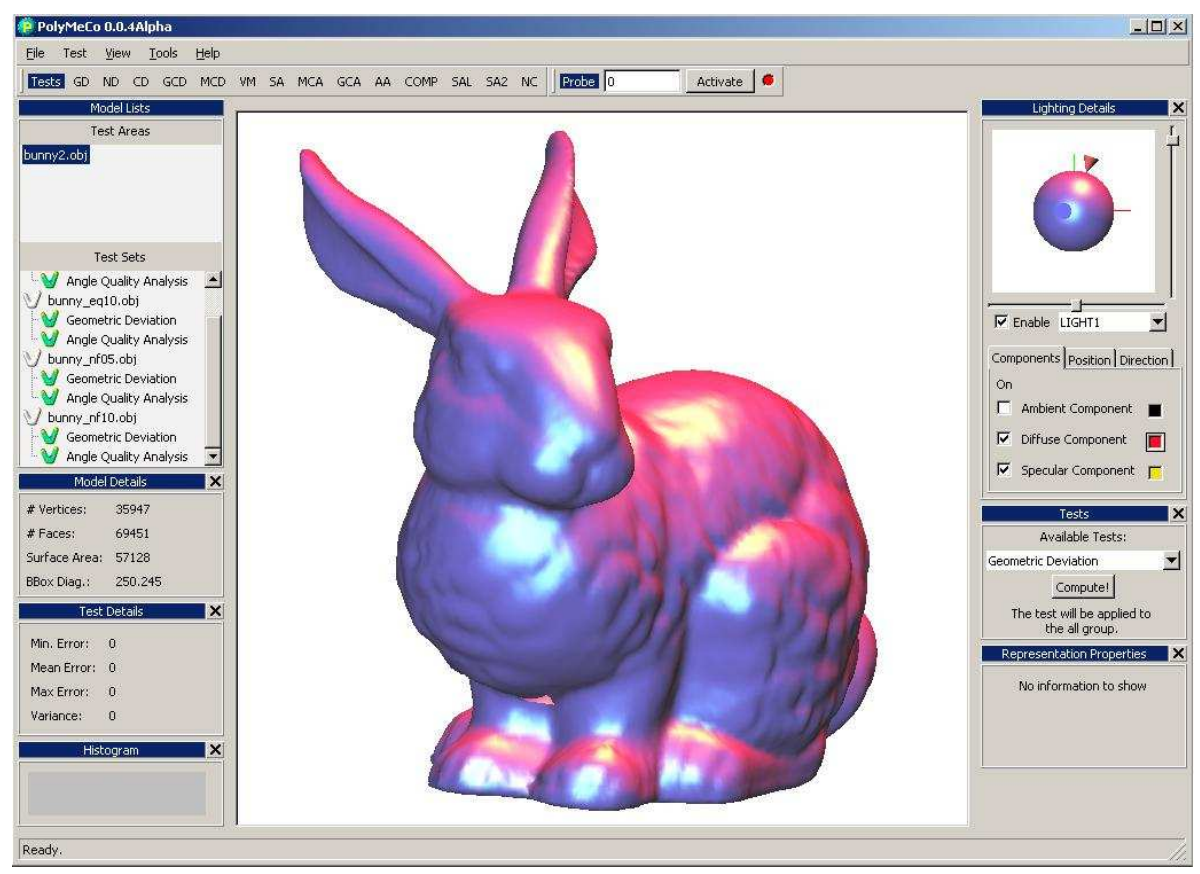

Figure 1: PolyMeCo's user interface.

PolyMeCo ${ }^{12}$ is a tool under development meant to provide an integrated environment where mesh analysis and comparison can be performed and the obtained results properly analysed (see figure 1), using different visualization options in a coordinated way. The main goal is not to provide new quality measures (although this tool seems an appropriate testbed) but a set of features which allow their usage in a systematic and efficient way.

\subsection{Computational Measures}

PolyMeCo provides several computational measures which support mesh analysis and comparison. Two types of measures are provided: intrinsic properties and difference measures.

Intrinsic* properties allow the measurement of a particular property of a mesh. PolyMeCo provides the computation of: Mean and Gaussian Curvatures using the method proposed by Meyers et al.; ${ }^{18}$ Triangle Quality by computing the minimum angle for each mesh face; ${ }^{19}$ Mesh Saliency, by using the method proposed by Lee et

\footnotetext{
${ }^{*}$ Here used in the sense of a property which is directly computed from a model data, irrespective of any other reference model; not in the Differential Geometry sense as in "intrinsic curvature".
} 
al. ${ }^{20}$ and Smoothness, by computing for each vertex its distance to the centroid of its direct neighbors (one-ring vertices).

Difference measures allow the comparison of properties between meshes. PolyMeCo provides the computation of: Geometric and Normal Deviations, using the method proposed by Roy et al..17 Mean and Gaussian Curvature Deviations; Visual Differences, using the method proposed by Karni et al. ${ }^{21}$ and enhancements proposed in Soerkine et al. ${ }^{22}$ and Composed Deviation, based on the results presented by Santos et al. ${ }^{9}$ and mixing the geometric and normal deviations using a blending factor which depends on the simplification ratio.

Notice that, although it might be an unusual task, PolyMeCo does not impose any constraint on the comparison between dissimilar models.

\subsection{Representations}

Data obtained by using any of the computational measures available in PolyMeCo can be presented to the user in several ways. The different representations provided can be grouped in three different categories: Numerical Values, 3D Representations and Statistical Representations.

\section{Numerical Values}

The easiest way to provide the user with feedback about the computed measures is by presenting some numerical values that characterize them (minimum, mean and maximum values, and variance) and/or the meshes for which they were computed (number of vertices, number of faces, bounding box diagonal and surface area).

\section{D Representations}

The analysis and comparison of mesh properties can be performed with the help of 3D models: either by a simple geometric model representation or by the superposition of any computational measure distribution along a model surface, i.e., the model is colored according to the value obtained for each vertex/face. This coloring can be performed by mapping the values range to a particular color scale. Currently, PolyMeCo provides the following color scales: rainbow (the most commonly used in the literature, but not always the better choice ${ }^{23}$ ), greyscale, linearized greyscale, blue to cyan, blue to yellow and linearized optimal. ${ }^{24}$ Another representation option for mesh analysis and comparison can be that of model superposition. A first choice is to render both models in a "solid" way, using different colors. This allows the perception of the areas where, for example, the processed model overlaps the reference model. A second alternative is to render the reference model with some degree of transparency, in order to let the user perceive the differences in volume between the two superimposed models. Finally, both models can be rendered in wireframe.
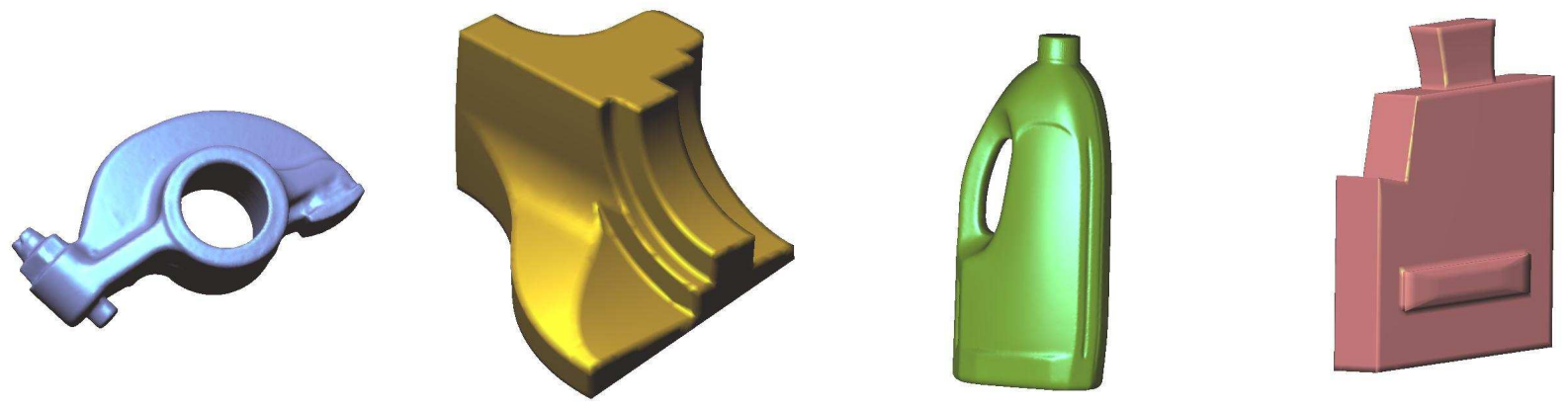

Figure 2: Models used for the evaluation. From left to right : RockerArm, FAnDisk, Flasche and BlockFS. 


\section{Statistical Representations}

Although model coloring gives a good idea of the distribution of a computed measure values along a model surface, showing where they occur, a global idea of the distribution characteristics can be difficult to obtain, due to the impossibility of viewing the whole surface simultaneously. Therefore, statistical representations may help to better understand and compare distributions of computed measures.

On PolyMeCo, histograms and boxplots are available. While the first provide information complementary to the one given by colored models (the user can, at a glance, get an idea of the global characteristics of the computed measure distribution), boxplots are very useful when comparing datasets, since they allow comparing and analysing the symmetries and ranges of the data, and detect the presence of outliers.

\subsection{Visualization and Interaction}

Visualization modes use the available representations in order to provide ways of better analysing and comparing mesh properties. Examples of visualization modes available in PolyMeCo are: Original vs Processed vs Colored Model, which allows the simultaneous view of both models being compared and a model colored according to the data obtained with a particular measure; Extended Results View, which shows a colored model, a histogram and a boxplot regarding some measure; and Features Comparison, which allows the visualization of data distributions obtained with the same computational measure for several processed models, and can get very useful in situations where the purpose is to study different processing algorithms and compare the obtained results. In order to allow better comparison using colored models, PolyMeCo allows using a common color map for all compared models, i.e., instead of using a color mapping for each model (maximum value obtained for that model mapped to the highest color) the maximum value obtained among all models is found and a common color map is used with this value mapped to the highest color. A similar feature also applies to histogram ranges, i.e., all histograms are drawn for the same value range.

\section{CASE STUDY}

For the evaluation of the QSlim and NSA simplification methods four polygonal models were chosen. Figure 2 shows those models and table 1 some details about their complexity.

Table 1: Number of vertices and faces for each of the used models.

\begin{tabular}{lrr} 
Models & \# Vertices & \# Faces \\
\hline FANDISK & 6,475 & 12,946 \\
\hline ROCKERARM & 40,177 & 80,354 \\
\hline FLASCHE & 42,762 & 85,524 \\
\hline BLOCKFS & 12,773 & 25,542
\end{tabular}

For each model and for three simplification levels (strong, moderate and light), a simplified model was created using QSlim and another using NSA. Figure 3 shows the complete set for the FANDISK model.

The following comparisons were carried out using PolyMeCo alpha version $0.0 .5 \mathrm{~b}^{\dagger}$.

\subsection{Preliminary Analysis}

First, a preliminary analysis was performed regarding the bounding box features and the surface area of the simplified models, comparing them with those of the original models. In particular, for the FANDISK and BLOCKFS models some important surface area differences were found for the models simplified using QSlim, which are related to some surface anomalies that will be analysed later.

A comparative visual analysis of volume was also performed by rendering the original model and each simplified version simultaneously (i.e., superimposed). Figure 4 shows this for two simplified versions of RocKERARM. The original model is rendered in red (with the option of changing its transparency level) and the simplified version in blue, thus allowing a perception of the areas where one of the models is over the other. For all simplified models no significant volume differences were found. 

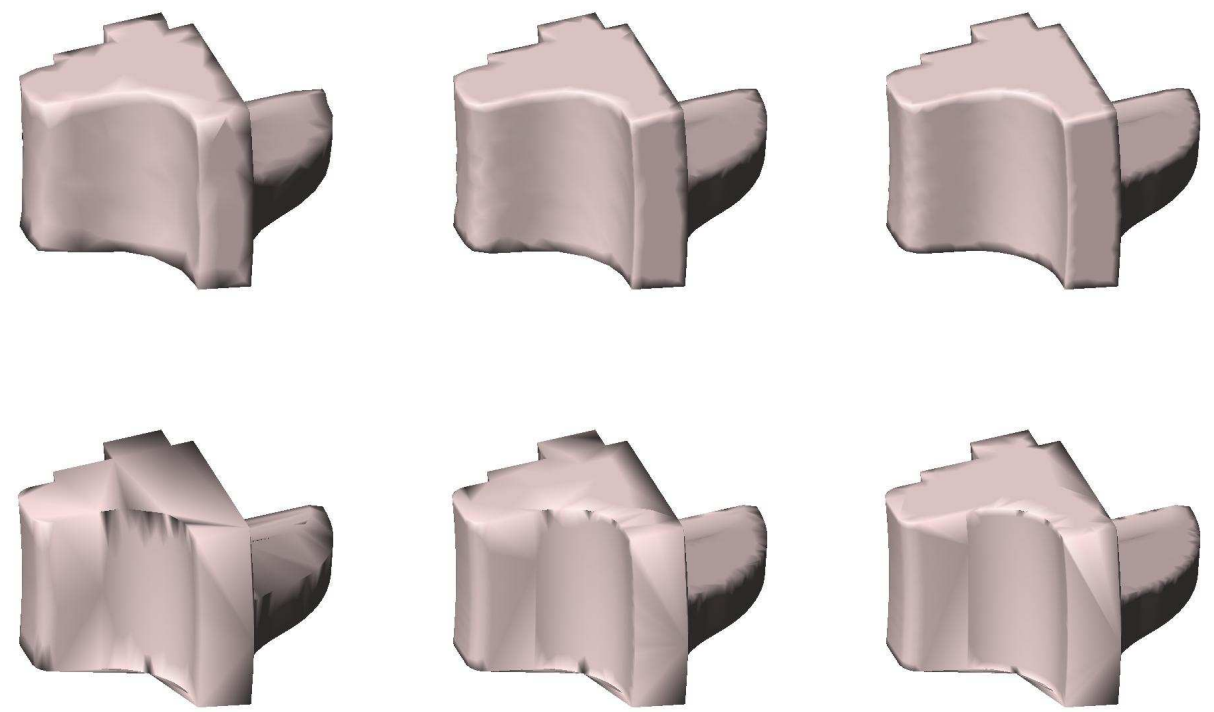

Figure 3: Simplified versions for the FANDisk model. Top, strongly, moderately and lightly simplified versions obtained with NSA; bottom, the corresponding versions obtained with QSlim.
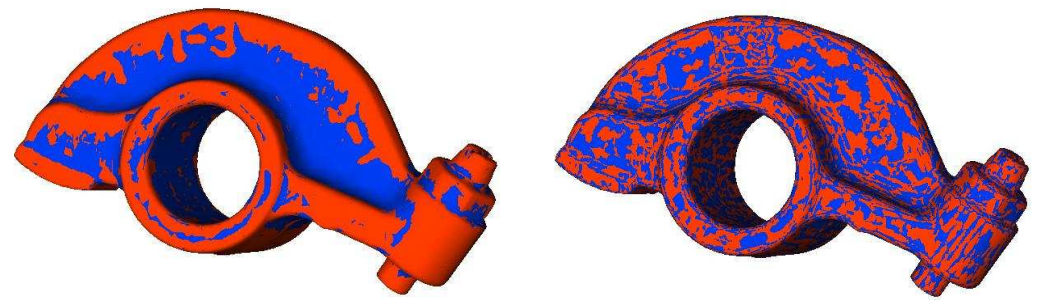

Figure 4: Comparative visual analysis of volume differences between the original model and the simplified versions RockerArm_5908_NSA (left) and RockerArm_5908_QS (right). Red areas correspond to the RockERARM model and blue areas to the simplified model.

Proper illumination can also help users analyse a particular mesh and this has been used in sophisticated ways in the automobile industry. ${ }^{25}$ As a first step towards this kind of analysis, PolyMeCo supports adding more point light sources and changing their properties (position and color of components), thus allowing the examination of surfaces in more detail. This kind of analysis is more effective when interactively repositioning the light sources which can also be done in PolyMeCo (although with some limitations depending on the complexity of the viewed mesh). In figure 5 some of the obtained views are presented; notice, on the left, how several artifacts are noticeable on the surface of the version simplified with QSlim and how, on the right, the surface highlights look better defined in the model simplified with QSlim.

\subsection{Analysis and Comparison using Computational Measures}

From the set of computational measures available in PolyMeCo the following were chosen for this case study: Geometric and Normal Deviations, Composed Deviation, Smoothness Analysis and Minimum Angle Analysis. In table 2 we present the mean values obtained for the different metrics when applied to the simplified versions of the chosen meshes. Note that each mesh name includes the number of faces and the used simplification

\footnotetext{
${ }^{\dagger} \mathrm{A}$ version of PolyMeCo for test purposes is available in http://www.ieeta.pt/polymeco
} 

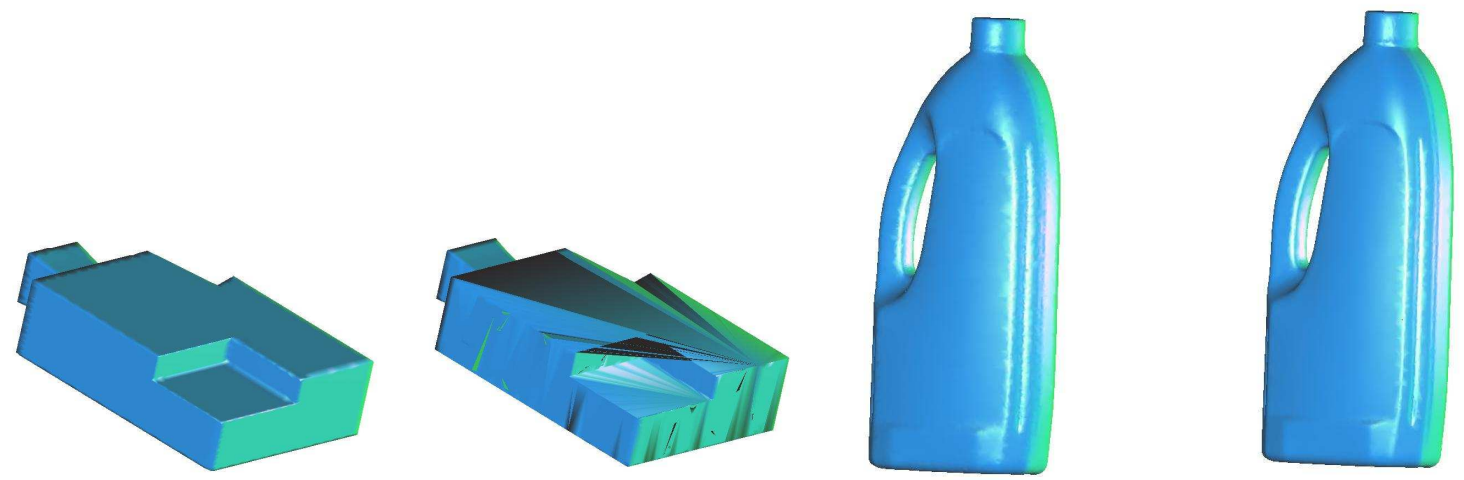

Figure 5: Models viewed after customising light source properties. From left to right: Blockfs_13040_NSA, Blockfs_13040_QS, Flasche_25615_NSA and Flasche_25615_QS
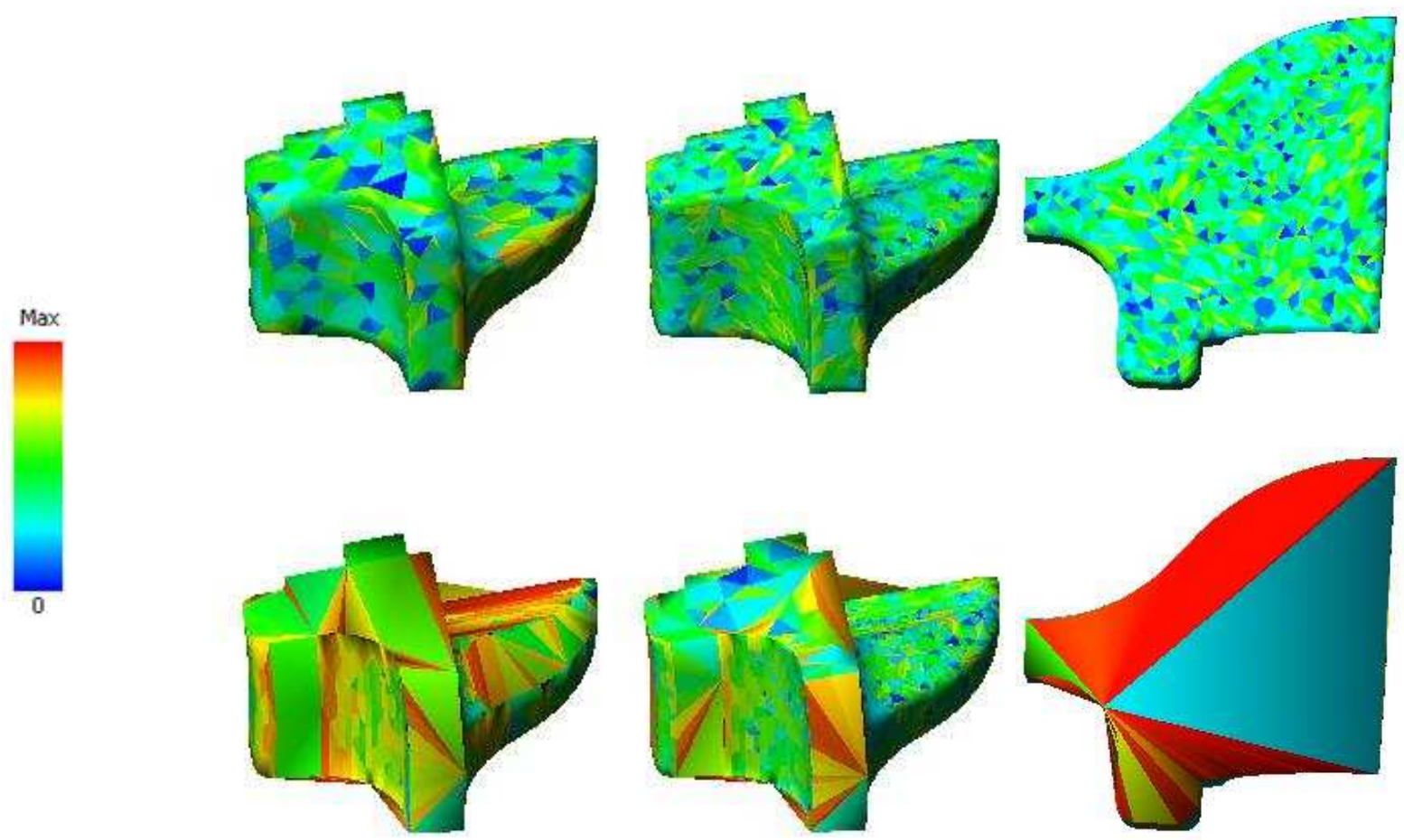

Figure 6: Colored models depicting the distribution of the values obtained with the Minimum Angle Analysis metric for the FANDISK model. Top, strongly, moderately and lightly simplified versions obtained with NSA; bottom, the corresponding versions obtained with QSlim. Areas in red are composed of flat or needle triangles.

algorithm. For example, Fandisk_7000_NSA is the simplified mesh of FANDISK with 7000 faces generated by NSA, and Fandisk_7000_QS is the simplified mesh of FANDISK with 7000 faces generated by QSlim.

For the FANDISK model in table 2, we can see that NSA produces better results than QSlim algorithm for all metrics, with the exception of Geometric Deviation and Minimum Angle Analysis for light simplifications. Figure 6 shows some models colored according to the results obtained using the Angle Analysis metric (the minimum angle value is better when it is closer to $60^{\circ}$ ). Notice how the bottom models (created using QSlim) exhibit a larger amount of irregular triangles (with at least one small angle) which results, for example, in very 
Table 2: Evaluating the simplified models created by QSlim and NSA using different metrics; mean values are presented.

\begin{tabular}{|c|c|c|c|c|c|}
\hline Meshes & Geom. Dev. & Normal Dev. & Comp. Dev. & Smoothness & Minimum Angle \\
\hline Fandisk_7000_NSA & $1.65 \mathrm{E}-4$ & $3.44 \mathrm{E}-2$ & $4.66 \mathrm{E}-2$ & $1.47 \mathrm{E}-5$ & 36.72 \\
\hline Fandisk_7000_QS & $8.32 \mathrm{E}-7$ & $2.08 \mathrm{E}-1$ & $9.74 \mathrm{E}-2$ & $1.61 \mathrm{E}-3$ & 38.88 \\
\hline Fandisk_3872_NSA & $3.65 \mathrm{E}-4$ & $6.21 \mathrm{E}-2$ & $5.59 \mathrm{E}-2$ & $6.23 \mathrm{E}-5$ & 34.81 \\
\hline Fandisk_3872_QS & $9.83 \mathrm{E}-6$ & $2.60 \mathrm{E}-1$ & $1.37 \mathrm{E}-1$ & $2.51 \mathrm{E}-3$ & 31.77 \\
\hline Fandisk_1312_NSA & $1.22 \mathrm{E}-3$ & $1.28 \mathrm{E}-1$ & $8.72 \mathrm{E}-2$ & $1.61 \mathrm{E}-4$ & 30.89 \\
\hline Fandisk_1312_QS & $4.75 \mathrm{E}-5$ & $3.91 \mathrm{E}-1$ & $9.50 \mathrm{E}-2$ & $3.84 \mathrm{E}-3$ & 20.78 \\
\hline RockerArm_40568_NSA & $1.19 \mathrm{E}-2$ & $2.95 \mathrm{E}-2$ & $9.16 \mathrm{E}-2$ & $2.84 \mathrm{E}-4$ & 35.10 \\
\hline RockerArm_40568_QS & $1.93 \mathrm{E}-3$ & $3.68 \mathrm{E}-1$ & $6.99 \mathrm{E}-2$ & $2.22 \mathrm{E}-4$ & 36.60 \\
\hline RockerArm_20742_NSA & $2.35 \mathrm{E}-2$ & $4.81 \mathrm{E}-2$ & $1.03 \mathrm{E}-1$ & $5.79 \mathrm{E}-4$ & 30.70 \\
\hline RockerArm_20742_QS & $4.76 \mathrm{E}-3$ & $2.81 \mathrm{E}-2$ & $1.02 \mathrm{E}-1$ & $5.15 \mathrm{E}-3$ & 34.60 \\
\hline RockerArm_5908_NSA & $6.59 \mathrm{E}-2$ & $1.00 \mathrm{E}-1$ & $1.48 \mathrm{E}-1$ & $3.81 \mathrm{E}-3$ & 29.70 \\
\hline RockerArm_5908_QS & $1.12 \mathrm{E}-2$ & $6.13 \mathrm{E}-2$ & $1.05 \mathrm{E}-1$ & $1.60 \mathrm{E}-3$ & 28.10 \\
\hline "Flasche_46444_NSA & $3.41 \mathrm{E}-2$ & $3.85 \mathrm{E}-2$ & $1.03 \mathrm{E}-1$ & $2.45 \mathrm{E}-3$ & 37.63 \\
\hline Flasche_46444_QS & $8.18 \mathrm{E}-3$ & $3.17 \mathrm{E}-2$ & $5.63 \mathrm{E}-2$ & $6.54 \mathrm{E}-4$ & 38.67 \\
\hline Flasche_25615_NSA & $6.32 \mathrm{E}-2$ & $6.05 \mathrm{E}-2$ & $1.32 \mathrm{E}-1$ & $4.66 \mathrm{E}-3$ & 36.66 \\
\hline Flasche_25615_QS & $1.52 \mathrm{E}-2$ & $4.65 \mathrm{E}-2$ & $8.75 \mathrm{E}-2$ & $1.06 \mathrm{E}-3$ & 32.56 \\
\hline Flasche_3094_NSA & $3.70 \mathrm{E}-1$ & $1.56 \mathrm{E}-1$ & $1.25 \mathrm{E}-1$ & $3.10 \mathrm{E}-2$ & 32.19 \\
\hline Flasche_3094_QS & $4.61 \mathrm{E}-2$ & $1.10 \mathrm{E}-1$ & $8.94 \mathrm{E}-2$ & $2.71 \mathrm{E}-2$ & 24.72 \\
\hline "BlockFS_13040_NSA & $3.23 \mathrm{E}-4$ & $3.50 \mathrm{E}-2$ & $2.93 \mathrm{E}-2$ & $2.40 \mathrm{E}-4$ & 26.04 \\
\hline BlockFS_13040_QS & 0.00 & $2.19 \mathrm{E}-1$ & $6.15 \mathrm{E}-2$ & $9.01 \mathrm{E}-2$ & 19.65 \\
\hline BlockFS_7000_NSA & $1.16 \mathrm{E}-3$ & $6.05 \mathrm{E}-2$ & $2.36 \mathrm{E}-2$ & $5.10 \mathrm{E}-3$ & 23.44 \\
\hline BlockFS_7000_QS & $1.00 \mathrm{E}-6$ & $3.17 \mathrm{E}-1$ & $5.31 \mathrm{E}-2$ & $9.80 \mathrm{E}-2$ & 16.31 \\
\hline BlockFS_3684_NSA & $2.75 \mathrm{E}-3$ & $9.52 \mathrm{E}-2$ & $2.66 \mathrm{E}-2$ & $1.55 \mathrm{E}-3$ & 24.99 \\
\hline BlockFS_3684_QS & $1.00 \mathrm{E}-6$ & $3.41 \mathrm{E}-1$ & 3.69E-2 & $1.65 \mathrm{E}-1$ & 18.23 \\
\hline
\end{tabular}

odd triangulations at the base of the model.

For the RocKERARM model a different behavior is observed. QSlim produces better results than NSA for all the metrics with the exception of Minimum Angle Analysis and Normal Deviation for light simplification. Figure 7 shows some models colored according to the values obtained using the Geometric Deviation. It is clear that the model created using $N S A$ exhibits larger deviation values. Histograms are an alternative way of looking at these results. Figure 8 shows a detail of the RocKERARM model when evaluated using the Composed Deviation. Once again the model created using $N S A$ obtained poorer results.

QSlim also produces better results for the FLASCHE model (table 2) in all metrics except for the Minimum Angle Analysis regarding light simplification. Figure 9, on the left, shows two models colored according to the computed Normal Deviation. The model created with NSA exhibits a larger deviation towards the original; on the right it is possible to verify a poorer triangle quality (smaller angles) in the model created with QSlim.

Finally, for the BLOCKFS model, NSA produces better results with the exception of those obtained using Geometric Deviation. Figure 10 shows models colored (using a common color map) according to the Normal Deviation for all of the simplified models. It is clear that all models created with QSlim exhibit larger deviation, mainly due to some triangulation problems similar to those shown in figure 6 for the FANDISK model. Figure 11 presents one of PolyMeCo's visualization modes, Extended Results View, showing the results obtained for model BlockFS_3684_QS using the Composed Deviation. By observing the boxplot, on the top right corner, it is possible to verify the existence of several points (in blue) above the top whisker. These points represent outliers detected on the data. The colorscale was adjusted (by using the sliders below the histogram) in order to exclude those values, which enables a better visualization of the results. 

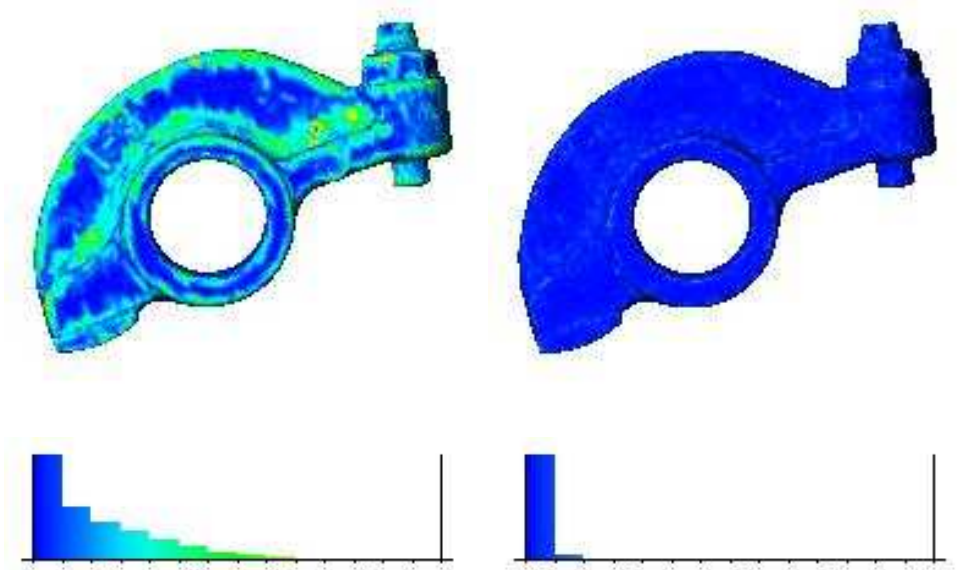

Figure 7: Colored models and histograms depicting the distribution obtained using the Geometric Deviation for the RockerArm_5908_NSA (left) and RockerArm_5908_QS (right) models.
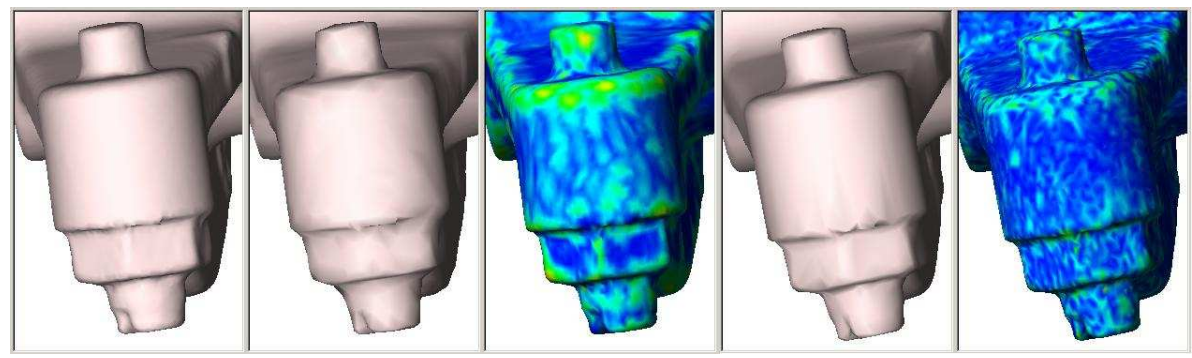

Figure 8: Composed Deviation results for the RoCKERARM model seen in greater detail. From left to right: original model, RockerArm_5908_NSA and deviation values, and RockerArm_5908_QS and deviation values.

\section{CONCLUSIONS}

From the analysis of the obtained results it is possible to conclude that QSlim always leads to simplified meshes with a lower mean Geometric Deviation when compared with the original mesh.

For the other metrics results depend on the nature of the mesh. For example, when dealing with meshes with planar regions (FANDISK and BLOCKFS) NSA produces better results than QSlim for all metrics except Geometric Deviation.

For models without planar regions QSlim produces, in general, better results for all metrics. However, for the same meshes, NSA yields better Minimum Angle Analysis results, as for the RockERARM model. For models with planar regions QSlim creates some surface artifacts due to the existence of vertices with a large number of incident edges, which explains why it normally yielded worst results for the Minimum Angle Analysis. This particularity can be a problem for visualization purposes and for finite element simulations where triangle quality is of extreme importance.

In fact, meshes created by QSlim have less geometric error than meshes created by $N S A$, but the visual quality of meshes created by QSlim is poorer when compared with the quality of the meshes created by $N S A$, in particular for models with planar regions as shown in figure 12.

PolyMeCo provided the possibility of analysing several simplified meshes using a large range of metrics, which allowed a more precise evaluation of the simplification algorithms. The Features Comparison visualization mode was particularly useful since it allowed comparisons (which is a harder task to perform when using just numerical values) using colored models and histograms (with common color maps and value ranges). The 

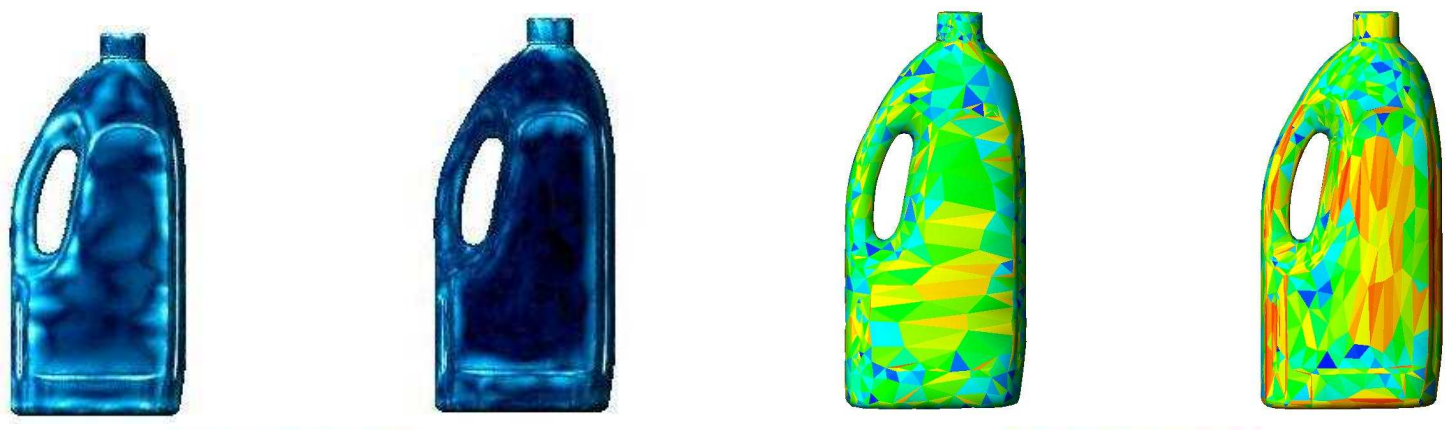

0

Max

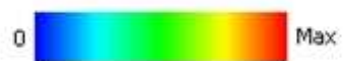

Figure 9: On the left, models colored using a blue-to-cyan colorscale according to the Normal Deviation values computed for models Flasche_3094_NSA and Flasche_3094_QS. On the right, Minimum Angle Analysis results for these same models.

blockss_3684_NSA.obj

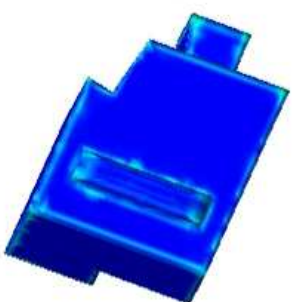

blockfs_3684_QS.obj

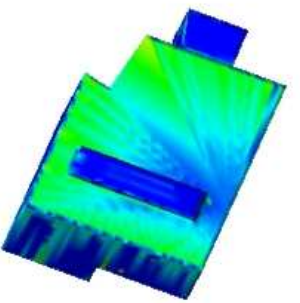

blockfs_ 7000 NSA.obj

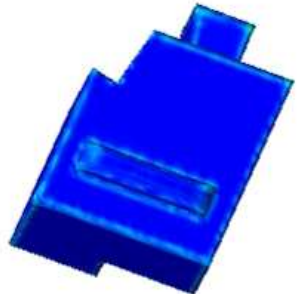

blockfs_7000_QS.obj

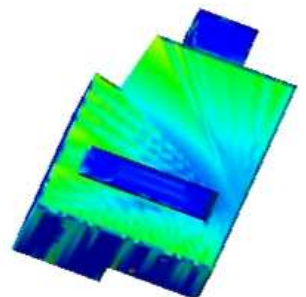

blockfs_13040_NSA.obj

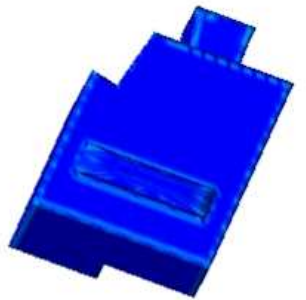

blockfs_13040_QS.obj

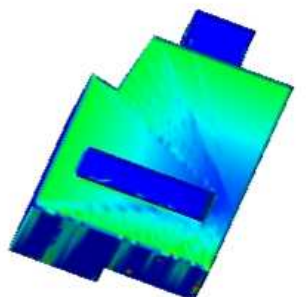

Figure 10: Colored models depicting the results obtained for the simplified versions of BLOCKFS using the Normal Deviation metric. A common color map is used for all models thus allowing direct comparison using the colored models. Top, strongly, moderately and lightly simplified models using $N S A$, bottom, the corresponding simplified models using QSlim.

available visualization options allowed a clearer understanding of the meaning of numerical values as well as the problems they are related to. A clear example is that of Angle Analysis values and the detection of odd triangles.

Having all models and data (from all the computed measures) simultaneously available in PolyMeCo allowed a more interactive analysis: it was possible to change between models and visualization modes while searching for the configurations which provided greater insight into the analysed data.

These features allowed a better understanding of the main characteristics of each algorithm. For instance, if the original model has a large number of planar regions, and visual quality is the most important criterion, QSlim might not be the algorithm of choice. 


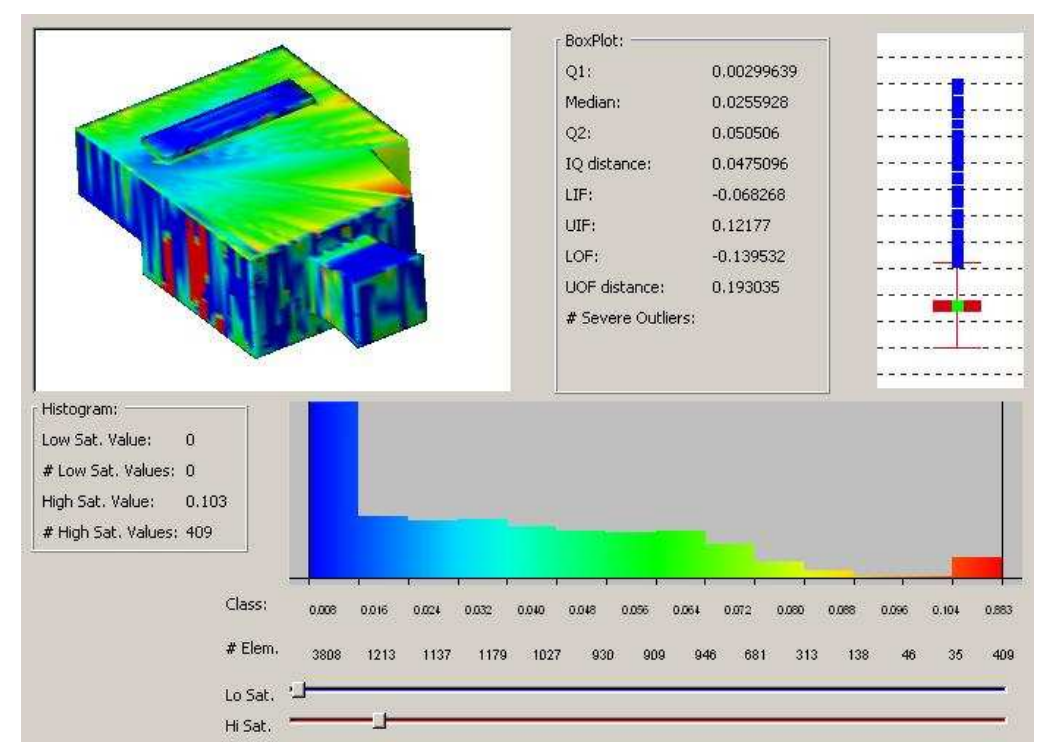

Figure 11: Extended results view for model BlockFS_3684_QS showing a colored model, a histogram and a boxplot for the results obtained with the Composed Deviation.

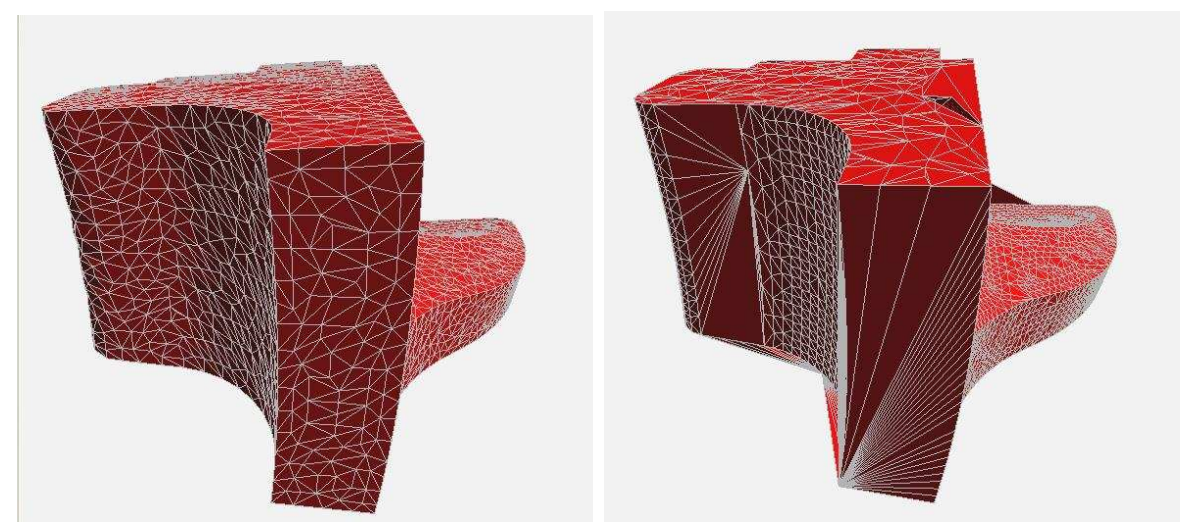

Figure 12: Fandisk mesh with 7000 faces created by NSA(left) and QSlim (right).

\section{ACKNOWLEDGMENTS}

The first author would like to thank research unit 127/94 IEETA of the University of Aveiro for the grant that supports his work.

The polygonal models used are courtesy of Cyberware, Stanford University and 3D Cafe.

\section{REFERENCES}

1. M. Levoy et al., "The digital Michelangelo project: 3D scanning of large statues," in Proc. SIGGRAPH 2000, pp. 131-144, 2000.

2. F. Losasso, I. Geoffrey, E. Guendelman, and R. Fedkiw, "Melting and burning solids into liquids and gases," IEEE Transactions on Visualization and Computer Graphics 12(3), pp. 343-352, 2006.

3. D. P. Luebke, "A developer's survey of polygonal simplification algorithms," IEEE Computer Graphics and Applications 21(3), pp. 24-35, 2001.

4. P. Cignoni, C. Montani, and R. Scopigno, "A comparison of mesh simplification algorithms," Computers and Graphics 22(1), pp. 37-54, 1998. 
5. B. E. Rogowitz and H. E. Rushmeier, "Are image quality metrics adequate to evaluate the quality of geometric objects?," in Proc. SPIE 2001 vol. 4299, Human Vision and Electronic Imaging VI, pp. 340-348, 2001.

6. B. Watson, A. Friedman, and A. McGaffey, "Measuring and predicting visual fidelity," in Proc. SIGGRAPH 2001, pp. 213-220, 2001.

7. S. Silva, B. S. Santos, J. Madeira, and C. Ferreira, "Comparing three methods for simplifying mesh models of the lungs: an observer test to assess perceived quality," in Proc. SPIE 2005 vol. 5749, Image Perception, Observer Performance, and Technology Assessment, pp. 99-110, 2005.

8. S. Silva, C. Ferreira, J. Madeira, and B. S. Santos, "Perceived quality of simplified polygonal meshes: Evaluation using observer studies," in Proc. Ibero-American Symposium in Computer Graphics SIACG06, pp. 169-178, (Santiago de Compostela, Spain), 2006.

9. B. S. Santos, S. Silva, C. Ferreira, and J. Madeira, "Comparison of methods for the simplification of mesh models of the lungs using quality indices and an observer study," in 3rd International Conference on Medical Information Visualization - Biomedical Visualization (MediVis'05), pp. 15-21, IEEE CS Press, 2005.

10. F. G. M. Silva and A. J. P. Gomes, "Normal-based simplification algorithm for meshes," in Proc. of Theory and Practice of Computer Graphics (TPCG'04), pp. 211-218, IEEE CS Press, 2004.

11. M. Garland and P. S. Heckbert, "Surface simplification using quadric error metrics," in Proc. SIGGRAPH 1997, pp. 209-216, 1997.

12. S. Silva, J. Madeira, and B. S. Santos, "Polymeco - a polygonal mesh comparison tool," in Proc. 9th International Conference on Information Visualization IV05, pp. 842-847, IEEE CS Press, 2005.

13. E. Puppo and R. Scopigno, "Simplification, LOD and multiresolution — principles and applications," Eurographics Tutorial Notes, 1997.

14. P. Cignoni, C. Rocchini, and R. Scopigno, "Metro: measuring error on simplified surfaces," Computer Graphics Forum 17(2), pp. 167-174, 1998.

15. L. Zhou and A. Pang, "Metrics and visualization tools for surface mesh comparison," in Proc. SPIE 2001 vol 4302, Visual Data Exploration and Analysis VIII, pp. 99-110, 2001.

16. N. Aspert, D. Santa-Cruz, and T. Ebrahimi, "MESH: Measuring errors between surfaces using the hausdorff distance," in Proc. IEEE Int. Conf. in Multimedia and Expo 2002, 1, pp. 705-708, (Lausanne, Switzerland), 2002.

17. M. Roy, S. Foufou, and F. Truchetet, "Mesh comparison using attribute deviation metric," Int. Journal of Image and Graphics 4(1), pp. 1-14, 2004.

18. M. Meyer, M. Desbrun, P. Schröder, and A. H. Barr, "Discrete differential-geometry operators for triangulated 2-manifolds," Visualization and Mathematics 3, pp. 34-57, 2002.

19. P. P. Pébay and T. J. T. J. Baker, "Analysis of triangle quality measures," Mathematics of Computation 72(244), pp. 1817-1839, 2003.

20. C. H. Lee, A. Varshney, and D. W. Jacobs, "Mesh saliency," in Proc. SIGGRAPH 2005, pp. 659-666, 2005.

21. Z. Karni and C. Gotsman, "Spectral compression of mesh geometry," in Proc. SIGGRAPH 2000, pp. 279286, 2000.

22. S. Sorkine, D. Cohen-Or, and S. Toledo, "High-pass quantization for mesh encoding," in Proc. Eurographics Symposium on Geometry Processing, pp. 42-51, 2003.

23. L. Bergman, B. Rogowitz, and L. Treinish, "A rule-based tool for assisting colormap selection," in 6th IEEE Visualization Conference, pp. 118-125, 1995.

24. H. Levkowitz and G. Herman, "Color scales for image data," IEEE Computer Graphics and Applications 12(1), pp. 72-80, 1992.

25. G. Sußner, G. Greiner, and S. Augustiniack, "Interactive examination of surface quality on car bodies," Computer-Aided Design 36, pp. 425-436, 2004. 